\title{
Первопринципное изучение реконструкций поверхности (001) полупроводников GaSb и InSb
}

\author{
(C) А.В. Бакулин ${ }^{1}$, С.Е. Кулькова ${ }^{1,2}$ \\ ${ }^{1}$ Институт фризики прочности и материаловедения Сибирского отделения Российской академии наук, \\ 634055 Томск, Россия \\ ${ }^{2}$ Национальный исследовательский Томский государственный университет, \\ 634050 Томск, Россия \\ E-mail: bakulin@ispms.tsc.ru
}

Поступила в Редакцию 3 февраля 2020 г.

В окончательной редакции 26 февраля 2020 г.

Принята к публикации 26 февраля 2020 г.

Методом проекционных присоединенных волн проведено изучение атомной и электронной структуры реконструкций с симметрией $(2 \times 4),(4 \times 2), c(4 \times 4)$ и $(4 \times 3)$ на поверхности $(001)$ полупроводников $\mathrm{GaSb}$ и InSb. Показано, что в пределе обогащения катионами на поверхности $\mathrm{GaSb}(001)$ стабильной является реконструкция $\beta 2(2 \times 4)$, тогда как $\alpha 2(2 \times 4)$ имеет наименьшую энергию в случае InSb. B пределе обогащения $\mathrm{Sb}$ стабильной найдена реконструкция $c(4 \times 4)$ с тремя димерами сурьмы. Вблизи стехиометрического состава на поверхности $\mathrm{GaSb}(001)$ стабильными являются структуры $\alpha(4 \times 3)$ и $\beta(4 \times 3)$, что согласуется с экспериментальными данными. Обсуждается электронная структура реконструкций $(4 \times 3)$ с наименьшей поверхностной энергией. Выявлено слабое влияние химического состава катионов на структуру и локализацию поверхностных состояний при формировании структур $(4 \times 3)$. Установлена корреляция между поверхностной энергией некоторых реконструкций $(4 \times 2)$ и $(2 \times 4)$ и разницей атомных радиусов катионов и анионов.

Ключевые слова: полупроводники $\mathrm{A}^{\mathrm{III}} \mathrm{B}^{\mathrm{V}}$, поверхность (001), реконструкция поверхности, электронная структура.

DOI: 10.21883/FTP.2020.07.49503.9363

\section{1. Введение}

Бинарные полупроводниковые соединения на основе элементов III и V групп являются важными структурными компонентами современной полупроводниковой индустрии. Во многих микро- и оптоэлектронных приборах и устройствах применяются гетероструктуры InAs/GaSb, InAs/GaIn и др., при этом часто используются экстремально тонкие полупроводниковые пленки [1-4]. Атомная структура полярных поверхностей полупроводников $\mathrm{A}^{\mathrm{III}} \mathrm{B}^{\mathrm{V}}$ представляет значительный научный и практический интерес, так как она непосредственно влияет на рост нано- и гетероструктур на этих поверхностях и на их электронные свойства. Понимание морфологии поверхности полупроводников $\mathrm{A}^{\mathrm{III}} \mathrm{B}^{\mathrm{V}}$ на атомном уровне является также принципиально важным для осуществления контролируемого роста тонких пленок металлов, сплавов, оксидов и других соединений, формирования интерфейсов [5,6], поскольку стерический и энергетический ландшафты поверхностной структуры определяют адсорбцию и десорбцию металлов и газов, a также их диффузию и обеспечивают условия для зарождения островков [7]. Повышенный интерес к структуре полярной поверхности $\mathrm{A}^{\mathrm{III}} \mathrm{B}^{\mathrm{V}}(001)$ обусловлен большим разнообразием поверхностных реконструкций, которые экспериментально наблюдаются в зависимости от состава поверхности и способа ее подготовки. Например, на поверхности $\mathrm{GaAs}(001)$ реконструкция с симметрией $c(4 \times 4)$ в случае высокой концентрации мышьяка превращается в $(2 \times 4) / c(2 \times 8)$ и далее в $(4 \times 2) / c(8 \times 2)$ при увеличении концентрации галлия. Кроме того, имеется большое разнообразие переходных структур с симметрией $(2 \times 1),(3 \times 1),(2 \times 3),(3 \times 6)$, $(2 \times 6),(4 \times 6),(6 \times 6)$ и др. [1,8-10].

Наиболее последовательно реконструкции поверхности $\mathrm{A}^{\mathrm{III}} \mathrm{B}^{\mathrm{V}}(001)$ теоретическими методами изучались в работе Шмидта [11]. В [11] приведены диаграммы стабильности поверхностных структур с симметрией $(2 \times 2),(2 \times 4),(4 \times 2)$ и $c(4 \times 4)$ для полупроводников $\mathrm{GaP}, \mathrm{InP}, \mathrm{GaAs}$ и InAs. Автор показал, что большинство экспериментальных результатов по реконструкциям поверхности (001) хорошо согласуется с данными, полученными методом псевдопотенциала. Некоторые реконструкции, такие как $\alpha 2(2 \times 4)$ и $\beta 2(2 \times 4)$, являются универсальными для рассмотренных соединений. Выявленные противоречия между теоретическими результатами и экспериментом автор [11] объяснил возможным влиянием способа приготовления поверхности. Кроме упомянутой выше работы вопрос о возможных реконструкциях поверхности $\mathrm{A}^{\mathrm{III}} \mathrm{B}^{\mathrm{V}}(001)$ рассматривался в многочисленных теоретических работах — например, [12-17]. Основной акцент в цитируемых работах делался на реконструкциях поверхности $\mathrm{GaAs}(001)$, тогда как поверхностные структуры в случаях InAs, GaP и InP изучены в гораздо меньшей степени.

Необходимо отметить, что вопрос о поверхностных структурах $\mathrm{GaSb}(001)$ и $\mathrm{InSb}(001)$ в литературе рассматривался в единичных работах [18-22], хотя эти 


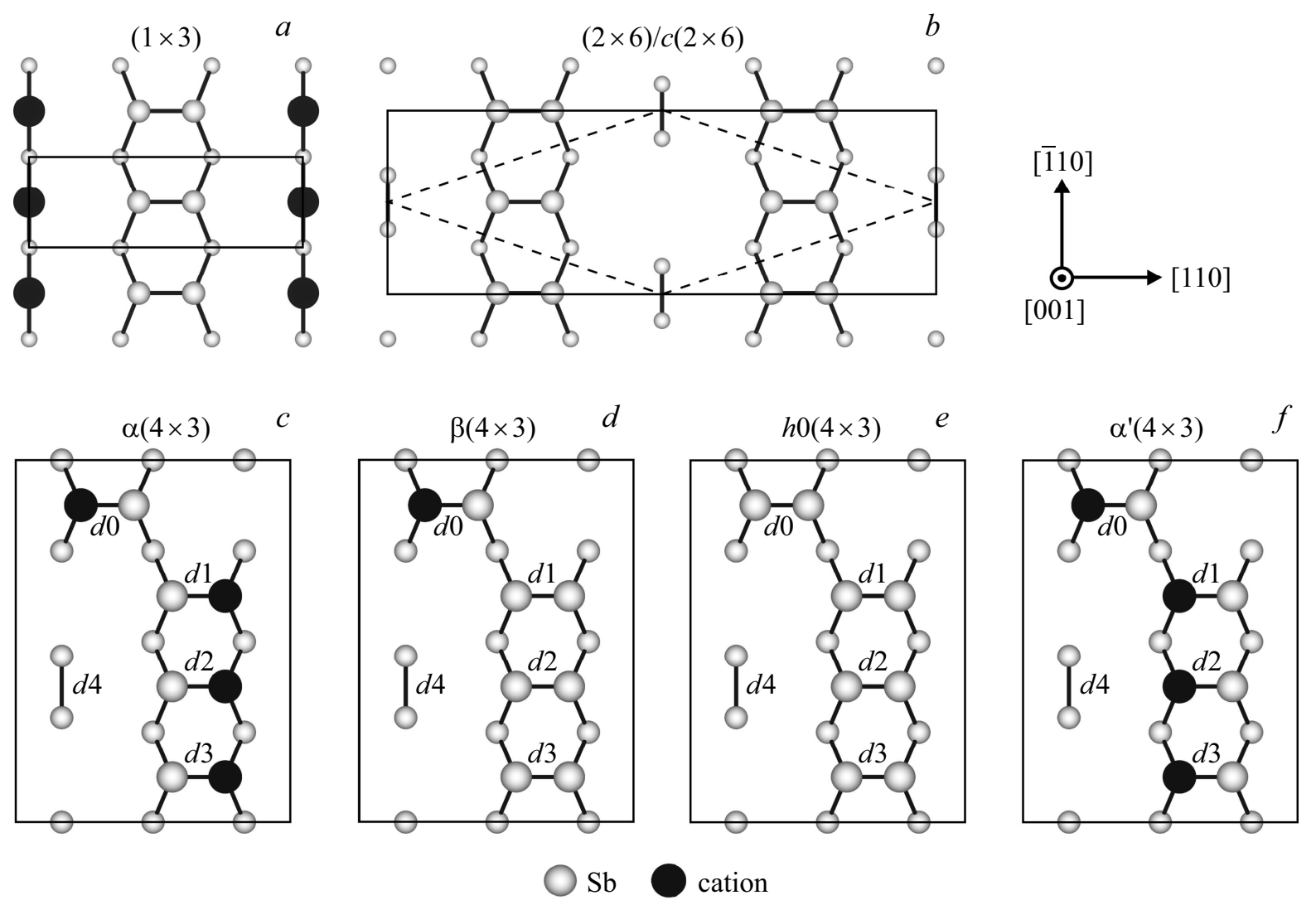

Рис. 1. Атомная структура поверхности $\mathrm{A}^{\mathrm{III}} \mathrm{Sb}(001)$ с различными реконструкциями: $a-(1 \times 3), b-(2 \times 6) / c(2 \times 6)$, $c-\alpha(4 \times 3), d-\beta(4 \times 3), e-h 0(4 \times 3), f-\alpha^{\prime}(4 \times 3)$. Катионы и атомы сурьмы показаны черными и белыми шариками соответственно.

соединения нашли широкое применение в современной полупроводниковой индустрии. В частности, они используются для создания светодиодов, работающих в инфракрасном спектре, туннельных диодов, сверхвысокочастотных транзисторов, датчиков Холла и других электронных компонентов. Для описания структуры поверхности $\mathrm{GaSb}(001)$ предлагались различные модели на основе экспериментов. Так, в работе [18] на поверхности $\mathrm{GaSb}(001)$ экспериментально наблюдалась слабо различимая фаза $(1 \times 3)$, на основе которой была предложена модель реконструкции, представленная на рис. $1, a$, со степенью покрытия галлием, равной $1 / 3$ монослоя (ML). Позднее в работе [19] на поверхности $\mathrm{GaSb}(001)$ были экспериментально обнаружены две реконструкции, с симметрией $(1 \times 3)$ и $c(2 \times 6)$. Необходимо отметить, что в обеих работах $[18,19]$ наблюдались одинаковые структуры с симметрией $(1 \times 3)$, а структура $c(2 \times 10)$ в [19] была обнаружена для экстремально высоких концентраций сурьмы (степень покрытия $\mathrm{Sb}>2 \mathrm{ML}$ ). Также в работе [19] на основе данных дифракции быстрых электронов и фотоэмиссионных исследований была предложена атомная модель реконструкции с симметрией $c(2 \times 6)$ (рис. $1, b)$. Поскольку данная поверхностная структура может также описываться прямоугольной поверхностной ячейкой $(2 \times 6)$, то на рис. $1, b$ она обо- значена как $(2 \times 6) / c(2 \times 6)$. Видно, что данная реконструкция подобна реконструкции $(1 \times 3)$, но отсутствие атомов галлия в поверхностном слое и несимметричное расположение димеров сурьмы приводит к понижению симметрии. В работе [20] показано, что при тех же условиях эксперимента, что и в [19], на поверхности $\mathrm{GaSb}(001)$ формируются две реконструкции с симметрией $(4 \times 3)$, получившие название $\alpha$ и $\beta$ (рис. $1, c, d)$. Видно, что в отличие от двух упомянутых выше структур они характеризуются наличием смешанных димеров $\mathrm{Ga}-\mathrm{Sb}$. Кроме того, в [20] было показано, что реконструкции $\alpha(4 \times 3)$ и $\beta(4 \times 3)$ формируются также на поверхности $\mathrm{AlSb}(001)$. Отметим, что предложенная в [20] реконструкция $\alpha(4 \times 3)$ имела три смешанных димера $(d 1-d 3$ на рис. $1, c)$, направленных в одну сторону, а отстоящий димер $\mathrm{Ga}(\mathrm{Al})-\mathrm{Sb}(d 0)$ был ориентирован в противоположную сторону. В этой же работе методом псевдопотенциала в рамках теории функционала электронной плотности с использованием приближения локальной плотности для обменно-корреляционного функционала авторы моделировали результаты исследования методом сканирующей туннельной микроскопии (СТМ) предложенных структур. Сопоставление теоретических и экспериментальных результатов СТМ показало их согласие. Однако в упомянутых выше работах не приво- 
дились поверхностные энергии реконструкций и не рассматривались их электронные энергетические спектры.

В настоящее время имеются лишь единичные теоретические работы [21,22], содержащие диаграммы стабильности, полученные на основе первопринципных расчетов поверхностных энергий ряда реконструкций $\mathrm{GaSb}(001)$ и $\mathrm{InSb}(001)$. В работе [21] в рамках приближения локальной плотности для обменно-корреляционного функционала с использование норм-сохраняющих псевдопотенциалов было показано, что реконструкция $\alpha(4 \times 3)$ явля-

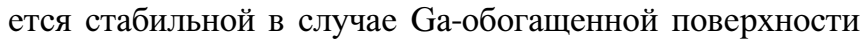
$\mathrm{GaSb}(001)$ и на поверхности стехиометрического состава, тогда как в $\mathrm{Sb}$-обогащенной области наименышую поверхностную энергию имеет реконструкция $\beta(4 \times 3)$. В пределе высоких концентраций сурьмы стабильной является структура $h 0(4 \times 3)$, атомная структура показана на рис. $1, e$. Отметим, что последняя реконструкция была предложена на основе структуры $\gamma(4 \times 3)$, наблюдаемой экспериментально на поверхности $\mathrm{AlSb}(001)$ [20]. Таким образом, согласно результатам работы [21], по мере увеличения концентрации $\mathrm{Sb}$ на поверхности $\mathrm{GaSb}(001)$ стабильными являются следующие реконструкции: $\alpha(4 \times 3) \rightarrow \beta(4 \times 3) \rightarrow h 0(4 \times 3)$. В [21] было отмечено, что в $\mathrm{Sb}$-обогащенной области реконструкции $\alpha, \beta, h 0, h 2, h 3$ с симметрией $(4 \times 3)$ имеют близкие поверхностные энергии, что указывает на возможность их сосуществования. Отметим, что структуры $h 0, h 2, h 3$ различаются количеством смешанных димеров на поверхности. В то же время в работе [21] рассматривалась лишь единственная реконструкция с симметрией $(2 \times 4)$, а именно $\beta 2(2 \times 4)$. Расчеты показали, что она является стабильной при экстремально высоких концентрациях галлия. В данной работе были также рассчитаны электронные энергетические спектры трех реконструкций $(4 \times 3)$ с наименьшей поверхностной энергией.

Реконструкции поверхности (001) полупроводников $\mathrm{GaSb}$ и AlSb изучались также в работе [22] с использованием ультрамягких псевдопотенциалов в рамках приближения локальной плотности для обменно-корреляционного функционала. В случае GaSb была подтверждена стабильность реконструкций $\alpha(4 \times 3)$ и $\beta(4 \times 3)$ для Ga-обогащенной поверхности и поверхности околостехиометрического состава, тогда как для $\mathrm{Sb}$-обогащенной поверхности наименьшую поверхностную энергию имеет структура $\gamma(4 \times 3)$. Однако рассчитанная разница в поверхностных энергиях для $h 0(4 \times 3)$ и $\gamma(4 \times 3)$ составляет лишь 4 мэВ/( $1 \times 1)$, что находится на пределе точности расчета. Необходимо отметить, что в [22] реконструкция $\alpha(4 \times 3)$, представленная на рис. $1, f$, имела несколько отличную структуру по сравнению с рассмотренной ранее в работах [20,21]. Как видно из рис. $1, f$, данная структура, которую в дальнейшем будем называть $\alpha^{\prime}(4 \times 3)$, отличается от оригинальной $\alpha(4 \times 3)$ ориентацией смешанных димеров $d 1-d 3$. Необходимо отметить, что в [22] поверхностные диаграммы стабильности для $\mathrm{GaSb}(001)$ и $\operatorname{InSb}(001)$ практически не отличаются друг от друга. Наблюдались лишь незначительные отличия для высокоэнергетических структур с симметрией $c(2 \times 10)$, которые в большом количестве рассматривались в данной работе, но ни одна из них не была найдена стабильной. Кроме того, реконструкции $(2 \times 4)$ и $(4 \times 2)$ в [22] не рассматривались, что не позволяет сравнить их поверхностные энергии с изученными реконструкциями $(4 \times 3)$.

В работе [23] наряду с исследованиями методом сканирующей туннельной микроскопии поверхности $\mathrm{InSb}(001)$ проводились первопринципные расчеты атомной структуры двух реконструкций: $(4 \times 2)$ и $c(8 \times 2)$. Авторы утверждают, что эти реконструкции являются стабильными и с точностью до погрешности расчета имеют одинаковые поверхностные энергии. Последнее находится в противоречии с экспериментальными данными [24-27], поскольку реконструкции $(4 \times 2)$ на поверхности $\operatorname{InSb}(001)$, в отличие от, например, $\operatorname{InAs}(001)$ [26,28], не наблюдались. Авторы [23] объяснили это частичной занятостью позиций поверхностных атомов, которая приводит к тому, что при комнатных температурах атомы могут совершать быстрые перескоки между соседними позициями и измерительный прибор регистрирует один и тот же атом в обеих позициях. При низких температурах такие перескоки редки, и эксперимент показывает поверхность с более низкой симметрией. Последнее имеет место и в случае теоретических расчетов. Отметим, что аналогичное обоснование использовалось в работе [29] для объяснения несоответствия между теоретической моделью и наблюдаемой экспериментально структурой $\operatorname{InAs}(001)-\beta 3^{\prime}(4 \times 2)$.

Таким образом, из приведенного выше обзора теоретических работ видно, что в настоящее время имеются немногочисленные работы, в которых в рамках одного метода проводилось сопоставление поверхностных энергий возможных реконструкций на поверхности (001) полупроводников $\mathrm{GaSb}$ и $\mathrm{InSb}$, в том числе со структурой $(2 \times 4)$ и $(4 \times 2)$. Напомним, что именно такие реконструкции были найдены стабильными на поверхности (001) полупроводников $\mathrm{GaP}, \mathrm{InP}, \mathrm{GaAs}$ и InAs [11-17]. Как было показано в работах [11,17], химический состав катионов достаточно слабо влияет на стабильность основных реконструкций, а анионы оказывают влияние в основном на поверхностную энергию реконструкции $\zeta(4 \times 2)$. В частности, реконструкция $\xi(4 \times 2)$ не являлась стабильной в случаях $\mathrm{GaP}(001)$ и $\operatorname{InP}(001)$, но она имела наименьшую поверхностную энергию в катион-обогащенной области на поверхности $\operatorname{GaAs}(001)$ и $\operatorname{InAs}(001)$.

В этой связи целью настоящей работы является сравнительное изучение реконструкций поверхности (001) соединений $\mathrm{GaSb}$ и $\mathrm{InSb}$ в рамках единого подхода. Наряду с реконструкциями $(4 \times 3)$ рассматриваются „классические“ реконструкции $(2 \times 4)$ и $(4 \times 2)$, а также обсуждаются общие закономерности в формировании структур на поверхности $\mathrm{A}^{\mathrm{III}} \mathrm{B}^{\mathrm{V}}(001)$. 


\section{2. Метод расчета}

Расчет атомной и электронной структуры поверхности (001) полупроводников GaSb и InSb c реконструкциями проводился методом проекционных присоединенных волн (PAW) в плосковолновом базисе [30,31], реализованным программным комплексом VASP [32,33], с обобщенным градиентным приближением для обменнокорреляционного функционала в форме GGA-PBE [34]. Энергия обрезания для плоских волн из базисного набора составляла 500 эВ. Рассчитанные параметры решетки $\mathrm{GaSb}$ и $\mathrm{InSb}$, равные 6.236 и $6.651 \AA$ соответственно, на 2.1-2.6\% превышают экспериментальные значения (6.10 и $6.48 \AA$ [35]). Структура полупроводников $\mathrm{A}^{\mathrm{III}} \mathrm{B}^{\mathrm{V}}$ в направлении [001] представляет собой чередование атомных слоев катионов и анионов, поэтому поверхность (001) в зависимости от ее окончания представлялась 8- или 9-слойными пленками, разделенными промежутками вакуума $\sim 15 \AA$. Оборванные связи сурьмы на одной стороне пленок насыщались атомами псевдоводорода $\mathrm{H}^{*}$ с дробным зарядом $0.75 e$. Таким образом, эта сторона пленки имела объемоподобный вид [36]. Равновесная длина связи $\mathrm{Sb}-\mathrm{H}^{*}$ составляла $1.761 \AA$. Позиции атомов псевдоводорода и атомов двух ближайших слоев сурьмы и галлия/индия фиксировались, тогда как положения атомов других слоев релаксировали до достижения сил на атомах $\sim 0.01$ эВ/А, при этом на другой стороне пленки моделировались реконструкции. Интегрирование по зоне Бриллюэна проводилось с использованием сетки $k$-векторов $6 \times 3 \times 1,3 \times 6 \times 1$ и $3 \times 4 \times 1$, полученной по схеме Монхорста-Пака [37], для реконструкций с симметрией $(2 \times 4),(4 \times 2)$ и $(4 \times 3)$ соответственно, что обеспечивало плотность точек не менее $2700 \AA^{2}$. Релаксация атомной структуры проводилась с использованием динамики Ньютона.

Известно, что основное состояние поверхности определяется минимумом термодинамического потенциала:

$$
\Omega=U-T S-\sum_{i} \mu_{i} n_{i}
$$

где $U-$ полная энергия системы, $\mu_{i}$ и $n_{i}-$ химический потенциал и количество атомов компонента $i$, а $T$ и $S-$ температура и энтропия соответственно. Поскольку энтропийный член $T S$ дает незначительный вклад в $\Omega$, им можно пренебречь. Кроме того, его вклад частично компенсируется при расчете поверхностной энергии реконструкций относительно некоторой реперной структуры. Таким образом, изменение термодинамического потенциала определяется изменением химических потенциалов компонентов полупроводника $\mathrm{AB}$, при этом справедливо соотношение $\mu_{i} \leq \mu_{i}^{\text {bulk }}$. Необходимо также учитывать, что на поверхности химический потенциал соединения равен сумме потенциалов компонентов: $\mu_{\mathrm{AB}}=\mu_{\mathrm{A}}+\mu_{\mathrm{B}}$. Если поверхность находится в равнове-

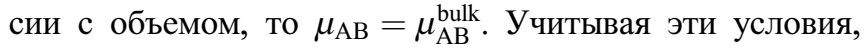

теплоту образования соединения можно представить в виде

$$
\begin{aligned}
-H_{\mathrm{AB}}^{f} & =\mu_{\mathrm{AB}}^{\text {bulk }}-\mu_{\mathrm{A}}^{\text {bulk }}-\mu_{\mathrm{B}}^{\text {bulk }} \\
& =\mu_{\mathrm{A}}-\mu_{\mathrm{A}}^{\text {bulk }}+\mu_{\mathrm{B}}-\mu_{\mathrm{B}}^{\text {bulk }}=\Delta \mu_{\mathrm{A}}+\Delta \mu_{\mathrm{B}} .
\end{aligned}
$$

Из формулы (2) можно найти границы изменения химического потенциала компонента А на поверхности:

$$
-H_{\mathrm{AB}}^{f} \leq \Delta \mu_{\mathrm{A}} \leq 0
$$

Поверхностная энергия рассчитывалась по следующей формуле:

$$
\gamma+\gamma_{\mathrm{H}^{*}}=\frac{1}{S}\left[E-n_{\mathrm{A}} \mu_{\mathrm{A}}-n_{\mathrm{B}} \mu_{\mathrm{B}}\right]
$$

где $\gamma-$ поверхностная энергия реконструированной поверхности, $\gamma_{\mathrm{H}^{*}}$ - энергия поверхности, пассивированной атомами псевдоводорода $\mathrm{H}^{*}, n_{i}$ и $\mu_{i}-$ число атомов и химический потенциал компонентов А или В. Поскольку интерес представляет расчет относительных поверхностных энергий, вторым слагаемым в левой части уравнения (4) можно пренебречь, так как оно имеет одинаковое значение для всех реконструкций. Подставляя в (4) выражение для $\mu_{\mathrm{B}}$ и учитывая (2), получаем следующее выражение для поверхностной энергии:

$$
\gamma=\frac{1}{S}\left[E-n_{\mathrm{B}} \mu_{\mathrm{AB}}^{\text {bulk }}-\left(n_{\mathrm{A}}-n_{\mathrm{B}}\right) \mu_{\mathrm{A}}^{\text {bulk }}-\left(n_{\mathrm{A}}-n_{\mathrm{B}}\right) \Delta \mu_{\mathrm{A}}\right] .
$$

Из формулы (5) видно, что поверхностная энергия представляется линейной функцией одной переменной $-\Delta \mu_{\mathrm{A}}$. В случае соединений GaSb и InSb формула (5) позволяет построить диаграмму стабильности для любых реконструкций поверхности в зависимости от концентрации галлия или индия. Из (5) следует, что реконструкции, которые находятся внизу диаграммы, имеют наименьшую поверхностную энергию и являются энергетически стабильными для данного значения $\Delta \mu_{\mathrm{Ga}}\left(\Delta \mu_{\mathrm{In}}\right)$.

Для расчета электронной структуры стабильных реконструкций применялся метод, называемый DFT-1/2 или LDA-1/2, в рамках которого используется нормальный потенциал Кона-Шема, но собственные значения соответствуют потенциалу частично ионизованного атома (занятость р-оболочки составляет 2.5 электрона вместо 3). Кулоновский потенциал анионов внутри некоторой сферы радиуса $r_{\text {cut }}$ также модифицируется путем умножения на функцию $\Theta=\left[1-\left(r / r_{\text {cut }}\right)^{n}\right]^{3}$, где $n-$ параметр, регулирующий скорость затухания потенциала на границе $r=r_{\text {cut }}$. Значение радиуса обрезания $r_{\text {cut }}$ выбирается так, чтобы оно соответствовало экстремуму функции ширины запрещенной щели $E_{g}$, а показатель $n$ должен быть как можно больше. На практике, как правило, выбирают $n=8$, но для ковалентных или слабо ионных соединений значение может быть увеличено 

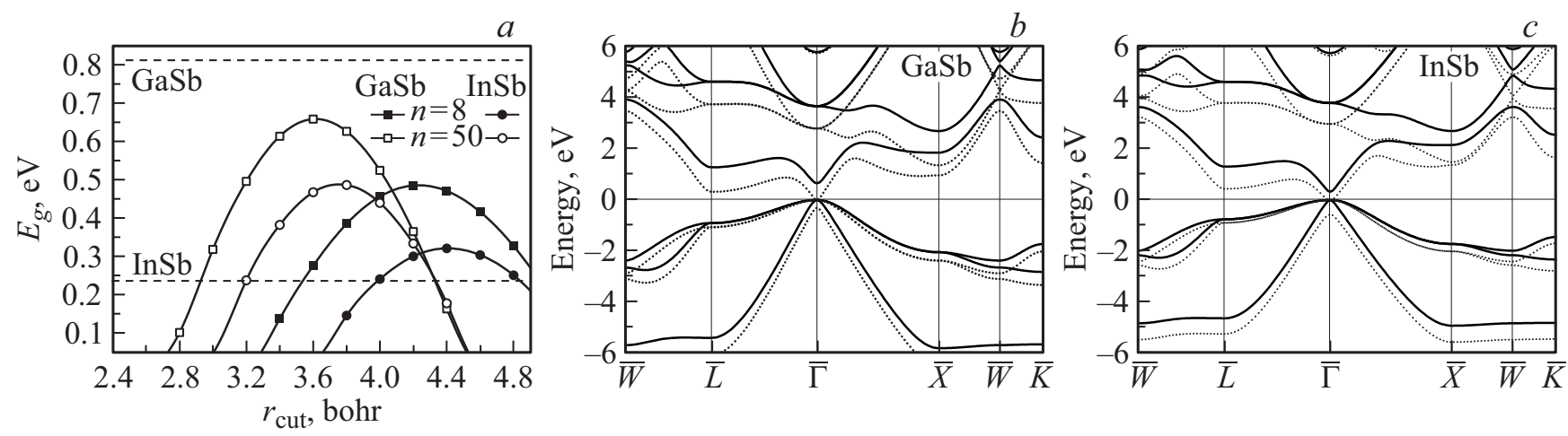

Рис. 2. Ширина запрещенной щели в объемных полупроводниках $\mathrm{GaSb}$ и $\mathrm{InSb}$ в зависимости от радиуса обрезания $r_{\text {cut }}$ и показателя степени $n(a) ;(b, c)$ - электронные энергетические спектры, рассчитанные в рамках PAW-PBE (пунктирные линии) и PAW-PBE-1/2 (сплошные линии).
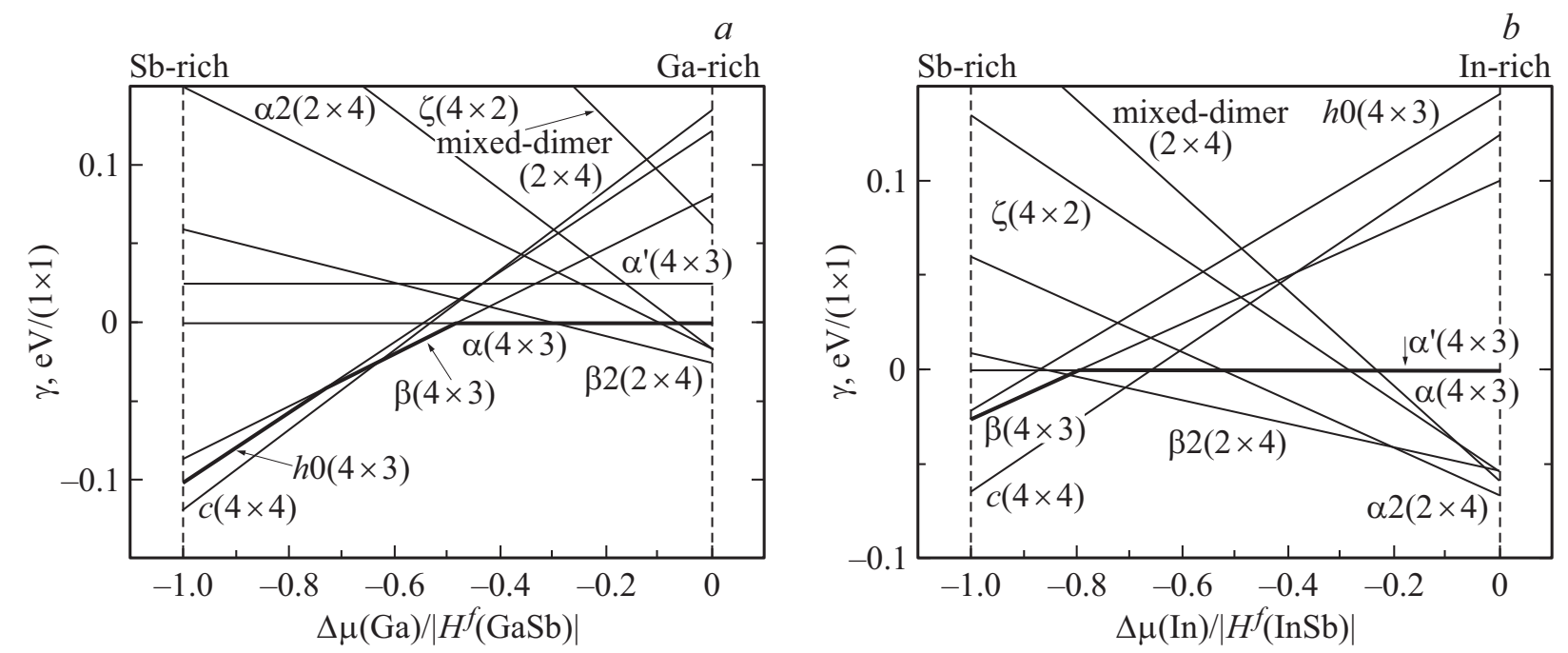

Рис. 3. Зависимость поверхностной энергии в расчете на ячейку $(1 \times 1)$ от химического потенциала галлия (индия) для изученных поверхностных реконструкций $\operatorname{GaSb}(001)(a)$ и $\operatorname{InSb}(001)(b)$. Толстой линией выделены реконструкции $(4 \times 3)$ с наименьшей поверхностной энергией.

до 50 или даже еще больше, если не возникает проблем со сходимостью [38]. Больше деталей можно найти в работах [38-41]. Полученные значения ширины щели в объемных полупроводниках $\mathrm{GaSb}$ и InSb для $n=8$ и 50 в зависимости от $r_{\text {cut }}$ представлены на рис. $2, a$. Несмотря на то что в расчетах запрещенная щель в случае $\mathrm{GaSb}$ по-прежнему недооценивается, а для InSb переоценивается, структура электронных спектров воспроизводится гораздо лучше, чем в рамках обычного расчета PAW-PBE (рис. 2, $b, c)$ : имеется запрещенная щель и правильное вырождение зон в точке Г. Напомним, что расчет обычным методом PAW-PBE дает нулевую щель для обоих полупроводников [42], а использование метода гибридных функционалов значительно увеличивает время расчетов. Таким образом, в настоящей работе использовались следующие параметры: $r_{\text {cut }}=3.6 \mathrm{bohr}$, $n=50$ в случае $\mathrm{GaSb}$ и $r_{\text {cut }}=4.0 \mathrm{bohr}, n=8$ в случаe InSb.

\section{3. Результаты и обсуждение}

\section{1. Диаграммы стабильности}

На рис. 3 приведены рассчитанные диаграммы стабильности поверхностей $\mathrm{GaSb}(001)$ и $\operatorname{InSb}(001)$, при этом показаны только основные реконструкции $(2 \times 4)$, $(4 \times 2)$ и $(4 \times 3)$ с наименьшей поверхностной энергией. Отметим, что на этих диаграммах под реконструкцией $\alpha(4 \times 3)$ понимается именно оригинальная структура, предложенная в [20] и показанная на рис. 1, $c$. Из рис. 3, $a$ видно, что при высоких концентрациях галлия на поверхности $\mathrm{GaSb}(001)$ стабильной является реконструкция $\beta 2(2 \times 4)$ с тремя димерами сурьмы в поверхностном слое, а в пределе высоких концентраций сурьмы - реконструкция $c(4 \times 4)$. Отметим, что в работе [22] поверхностная энергия структуры $c(4 \times 4)$ в пределе обогащения анионами была лишь 
Таблица 1. Поверхностная энергия (в мэВ/(1×1)) рассмотренных реконструкций относительно структуры $\alpha(4 \times 3)$

\begin{tabular}{|c|c|c|c|c|}
\hline Соединение & \multicolumn{2}{|c|}{$\mathrm{GaSb}$} & \multicolumn{2}{|c|}{ InSb } \\
\hline Реконструкция & Обогащение $\mathrm{Sb}$ & Обогащение Ga & Обогащение $\mathrm{Sb}$ & Обогащение In \\
\hline$\alpha((4 \times 3)$ & 0 & 0 & 0 & 0 \\
\hline$\beta(4 \times 3)$ & $-87,-100[21],-74[22]$ & $81,80[21], 76[22]$ & -26 & 100 \\
\hline$h 0(4 \times 3)$ & $-102,-110[21],-83[22]$ & $122,120[21], 118[22]$ & -22 & 146 \\
\hline$\alpha(2 \times 4)$ & 175 & 6 & 78 & -48 \\
\hline$\alpha 2(2 \times 4)$ & 152 & -17 & 60 & -66 \\
\hline$\alpha 3(2 \times 4)$ & 326 & 74 & 161 & -29 \\
\hline$\beta(2 \times 4)$ & 95 & 11 & 52 & -11 \\
\hline$\beta 2(2 \times 4)$ & $59,80[21]$ & $-25,0[21]$ & 9 & -54 \\
\hline$(2 \times 4)$ mixed-dimer & 398 & 62 & 194 & -59 \\
\hline$(2 \times 4)$ top-Ga(In)-dimer & 517 & 96 & 301 & -14 \\
\hline$\beta(4 \times 2)$ & 318 & 66 & 225 & 36 \\
\hline$\beta 2(4 \times 2)$ & 307 & 54 & 213 & 24 \\
\hline$\beta 3(4 \times 2)$ & 364 & 112 & 266 & 76 \\
\hline$\beta 3^{\prime}(4 \times 2)$ & 356 & 104 & 210 & 21 \\
\hline$\xi(4 \times 2)$ & 236 & -17 & 135 & -54 \\
\hline$c(4 \times 4)$ & $-118,-84[22]$ & $135,142[22]$ & -65 & 124 \\
\hline
\end{tabular}

незначительно, на 3 мэВ/( $1 \times 1)$, выше, чем $\gamma(4 \times 3)$, и практически равной $h 0(4 \times 3)$. В настоящем расчете разница между энергиями $c(4 \times 4)$ и $h 0(4 \times 3)$ составляет $16 \mathrm{мэВ/(1 \times 1).}$

$\mathrm{B}$ пределе обогащения $\mathrm{Ga}$ реконструкции $\alpha 2(2 \times 4)$ и $\xi(4 \times 2)$ имеют практически равные энергии и отличаются на $8 \mathrm{мэB/(1 \times 1)}$ от $\beta 2(2 \times 4)$, тогда как энергия структуры $(2 \times 4)$ со смешанным димером (mixeddimer), которая была найдена стабильной в случае полупроводников GaP, InP, GaAs и InAs [11,16,17], выше на 87 мэВ/(1×1). Структура $\beta(4 \times 3)$ найдена стабильной в интервале изменения химического потенциала галлия от -0.63 до -0.48 , что согласуется с результатами работы [22]. Реконструкция $\alpha(4 \times 3)$ в пределе обогащения катиоонами имеет энергию на 17 мэВ/(1 $\times 1)$ выше, чем структуры $\alpha 2(2 \times 4)$ и $\zeta(4 \times 2)$. Отметим, что $\alpha 2(2 \times 4), \xi(4 \times 2)$ и ряд других структур $(4 \times 2)$ и $(2 \times 4)$ в работах [21,22] не рассматривались, за исключением $\beta 2(2 \times 4)$ в [21]. Кроме того, наши расчеты показали, что поверхностная энергия реконструкции $\alpha(4 \times 3)$ на 25 мэВ/(1 $\times 1)$ меньше, чем $\alpha^{\prime}(4 \times 3)$. Ранее эти две реконструкции в рамках одного подхода также не изучались.

Из рис. $3, b$ видно, что на поверхности $\operatorname{InSb}(001)$ структура $(2 \times 4)$ со смешанным димером и $\zeta(4 \times 2)$ имеют энергию на $7-12$ мэВ/( $1 \times 1)$ выше, чем $\alpha 2(2 \times 4)$. В то же время реконструкция $\xi(4 \times 2)$ имеет наименьшую поверхностную энергию среди структур с геометрией $(4 \times 2)$ для обоих полупроводников. Структура $\beta(4 \times 3)$ на поверхности $\operatorname{InSb}(001)$ не является стабильной вблизи стехиометрического состава, где меньшую энергию имеет реконструкция $\beta 2(2 \times 4)$, а при высоких концентрациях сурьмы ее энергия выше на 39 мэВ/(1 $\times 1)$, чем в случае реконструкции $c(4 \times 4)$. В отличие от поверхности $\mathrm{GaSb}(001)$ поверхностные энергии структур $\alpha(4 \times 3)$ и $\alpha^{\prime}(4 \times 3)$ в случае $\operatorname{InSb}(001)$ практически равны (разница меньше, чем точность расчета поверхностных энергий). Детальная информация о поверхностной энергии всех рассчитанных реконструкций относительно структуры $\alpha(4 \times 3)$ приведена в табл. 1.

В целом полученные диаграммы стабильности для $\mathrm{GaSb}(001)$ и $\mathrm{InSb}(001)$ существенно отличаются от рассчитанных ранее диаграмм для полупроводников GaAs, InAs, GaP, InP [11-17] (см. работы и ссылки в них). Рассчитанные нами диаграммы для структур с наименьшими поверхностными энергиями представлены на рис. 4. Авторы [21] предположили, что отличие поверхностных диаграмм GaAs и GaSb обусловлено большей на 1.1 эВ энергией когезии $\left(E_{\mathrm{coh}}\right) \mathrm{Sb}$ по сравнению с $\mathrm{GaSb}$, тогда как в случае As и GaAs наблюдается обратное соотношение и разница составляет 0.3 эВ. На этом основании они также объяснили бо́льшую стабильность реконструкции $\alpha(4 \times 3)$ в интервале энергий $-0.5<\Delta \mu(\mathrm{Ga}) /\left|H^{f}(\mathrm{GaSb})\right|<0$ по сравнению с

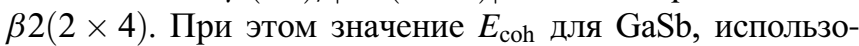
ванное в [21], было взято из работы [43]. Однако согласно данным других работ (табл. 2) энергия когезии $\mathrm{GaSb}$ оказывается практически в 2 раза больше. Из табл. 2 видно, что энергия когезии бинарных соединений практически всегда больше, чем их компонентов, поэтому такое объяснение не может считаться правильным.

Другое объяснение обнаруженным особенностям будет дано в следующем разделе, здесь же приведем несколько замечаний, касающихся различия теоретических диаграмм стабильности и экспериментально наблюдаемых структур. Реконструкция $c(4 \times 4)$ с тремя анионными димерами является самой анион-обогащенной структурой из рассмотренных нами. Данная структура $c(4 \times 4)$, как показано в ранних работах $[11,14,16,17]$ 
Таблица 2. Энергия когезии (в эВ/атом) элементов III и $\mathrm{V}$ групп и бинарных соединений на их основе $\left(\mathrm{A}^{\mathrm{III}} \mathrm{B}^{\mathrm{V}}\right)$

\begin{tabular}{c|c|c}
\hline $\begin{array}{c}\text { Катион } \rightarrow \\
\text { Анион } \downarrow\end{array}$ & Ga & In \\
\hline P 3.43 [44] & $3.81[44]$ & $2.52[44]$ \\
\hline & $3.56[46]$ & $3.40[45]$ \\
As 2.96[44] & $3.35[45]$ & $3.48[46]$ \\
& $3.26[46]$ & $3.25[45]$ \\
Sb 2.75 [44] & $3.03[45]$ & $3.10[46]$ \\
$2.6[43]$ & $2.96[46]$ & $2.90[45]$ \\
& $1.5[43]$ & $2.80[46]$
\end{tabular}

и ссылках в них, а также установлено в настоящем расчете, имеет наименьшую поверхностную энергию в пределе обогащения анионами во всех изученных полупроводниках $\mathrm{A}^{\mathrm{III}} \mathrm{B}^{\mathrm{V}}$. Поскольку методы из первых принципов позволяют рассчитывать поверхностные энергии уже сформировавшихся реконструкций, то противоречие теории и эксперимента (стабильность реконструкций $(2 \times 4)$, но формирование структур $(4 \times 3))$ может быть объяснено тем, что образование данных реконструкций ограничено другими факторами - например, кинетическими. Кинетика формирования поверхностных структур может быть оценена только с привлечением дополнительных теоретических методов. Например, метод подталкивающих упругих связей (nudged elastic band) [47] интенсивно используется в последние годы для оценки барьеров миграции и десорбции, а также энергии активации диффузии. Поскольку изучение диффузии на поверхности полупроводников является самостоятельной и достаточно трудоемкой задачей с точки зрения вычислительных затрат, оно будет проведено в последующей работе. Здесь же ограничимся только общими соображениями по этому поводу. Поскольку атомы фосфора очень легкие, они могут диффундировать по поверхности, при этом образование димеров фосфора является энергетически очень выгодным изза высокой энергии когезии фосфора (табл. 2). Хотя атомы мышьяка тяжелее атомов фосфора в 2.4 раза, они также могут считаться достаточно легкими, а чистый мышьяк может даже сублимировать [48], поэтому его миграция по поверхности достаточна вероятна. Это позволяет объяснить, почему экспериментально на поверхности (001) фосфидов и арсенидов галлия и индия наблюдается формирование структуры $c(4 \times 4)$ [49-53]. Так как атомы сурьмы в 1.6 и 3.9 раз тяжелее, чем атомы мышьяка и фосфора соответственно, их диффузия требует бо́льших температур и характеризуется меньшими скоростями. Этим может объясняться отсутствие реконструкции $c(4 \times 4)$ на поверхности $(001)$ полупроводников $\mathrm{GaSb}$ и $\mathrm{InSb}$, хотя расчеты показывают ее предпочтительность по сравнению с наблюдаемыми $\beta(4 \times 3)$ или $\gamma(4 \times 3) / h 0(4 \times 3)$. Аналогичные сообра- жения могут иметь место и при объяснении, почему реконструкции $(2 \times 4)$ и $(4 \times 2)$ не наблюдаются экспериментально. Поскольку симметрия реконструкций $(2 \times 4)$ и $(4 \times 2)$ выше, чем $(4 \times 3)$, они не могут сформироваться в условиях замедленной поверхностной диффузии. В пользу этой точки зрения косвенно свидетельствует также тот факт, что на поверхности $\operatorname{InSb}(001)$ экспериментально наблюдаются большие поверхностные структуры, в частности $(8 \times 2)$ [24,25]. Таким образом, если исключить из рассмотрения $c(4 \times 4),(2 \times 4)$ и $(4 \times 2)$, то на поверхности $\mathrm{GaSb}(001)$ при уменьшении содержания $\mathrm{Sb}$ должны формироваться следующие реконструкции: $h 0(4 \times 3) \rightarrow \beta(4 \times 3) \rightarrow \alpha(4 \times 3)$ (рис. $3, a)$. В случае $\operatorname{InSb}(001)$ стабильными являются только структуры $\beta(4 \times 3)$ и $\alpha(4 \times 3)$ (рис. $3, b)$. Отметим, что это согласуется с результатами ранних работ $[21,22]$ в случае $\mathrm{GaSb}(001)$, тогда для $\mathrm{InSb}(001)$ в [22] на небольшом интервале изменения химического потенциала сурьмы стабильной была найдена также структура $\gamma(4 \times 3)$. Заметим, что структуры $\gamma(4 \times 3)$ и $h 0(4 \times 3)$ различаются формой поверхностной ячейки: косоугольная в первом случае и прямоугольная во втором (см. рис. 1 в [22]). В работе [14] было показано на примере структуры $\beta 2-\mathrm{GaAs}(001)$ с симметрией $c(8 \times 2)$ и $(4 \times 2)$, что разница в поверхностных энергиях не превосходит $1.25 \mathrm{M} В /(1 \times 1)$ и не может считаться значимой. Аналогично разница в поверхностных энергиях структур $\gamma(4 \times 3)$ и $h 0(4 \times 3)$ в случаях $\mathrm{GaSb}(001)$ и $\mathrm{InSb}(001)$ составляет $4 \mathrm{MэB} /(1 \times 1)$ и $\sim 1$ мэВ/(1 $\times 1)$ [22] соответственно. Исходя из этих соображений, в настоящей работе мы рассматривали только реконструкцию $h 0(4 \times 3)$.

\section{2. Размерный эффект}

Возвращаясь к вопросу об общих тенденциях в диаграммах стабильности полярной поверхности (001) шести полупроводников $\mathrm{A}^{\mathrm{III}} \mathrm{B}^{\mathrm{V}}$, напомним, что в пределе обогащения катионами соединений $\mathrm{GaP}$ и InP структура $(2 \times 4)$ со смешанным димером стабильна в достаточно широком интервале изменений химического потенциала катиона (рис. $4, a, b)$, тогда как для GaAs и InAs лишь в пределе высоких концентраций катионов (рис. 4,c,d). Отметим, что лишь в работе [11] на поверхности $\mathrm{GaP}$ в пределе обогащения катионами стабильной была найдена реконструкция $(2 \times 4)$ с верхним Ga-димером (top-Ga-dimer). Однако в случаях $\mathrm{GaSb}$ и $\mathrm{InSb}$ структура $(2 \times 4)$ со смешанным димером не является стабильной (рис. 3). Структура $\xi(4 \times 2)$ была найдена стабильной лишь в случае полупроводников GaAs и InAs [11,14-16], при этом в последнем случае для очень узкого интервала изменения химического потенциала индия (рис. $4, d$ ).

Как отмечалось в [11], стабильность реконструкции $\xi(4 \times 2)$ может коррелировать с разницей в размерах анионов и катионов для конкретного полупроводника $\mathrm{A}^{\mathrm{III}} \mathrm{B}^{\mathrm{V}}$. Кроме того, смешанный димер на поверхности 
$a$
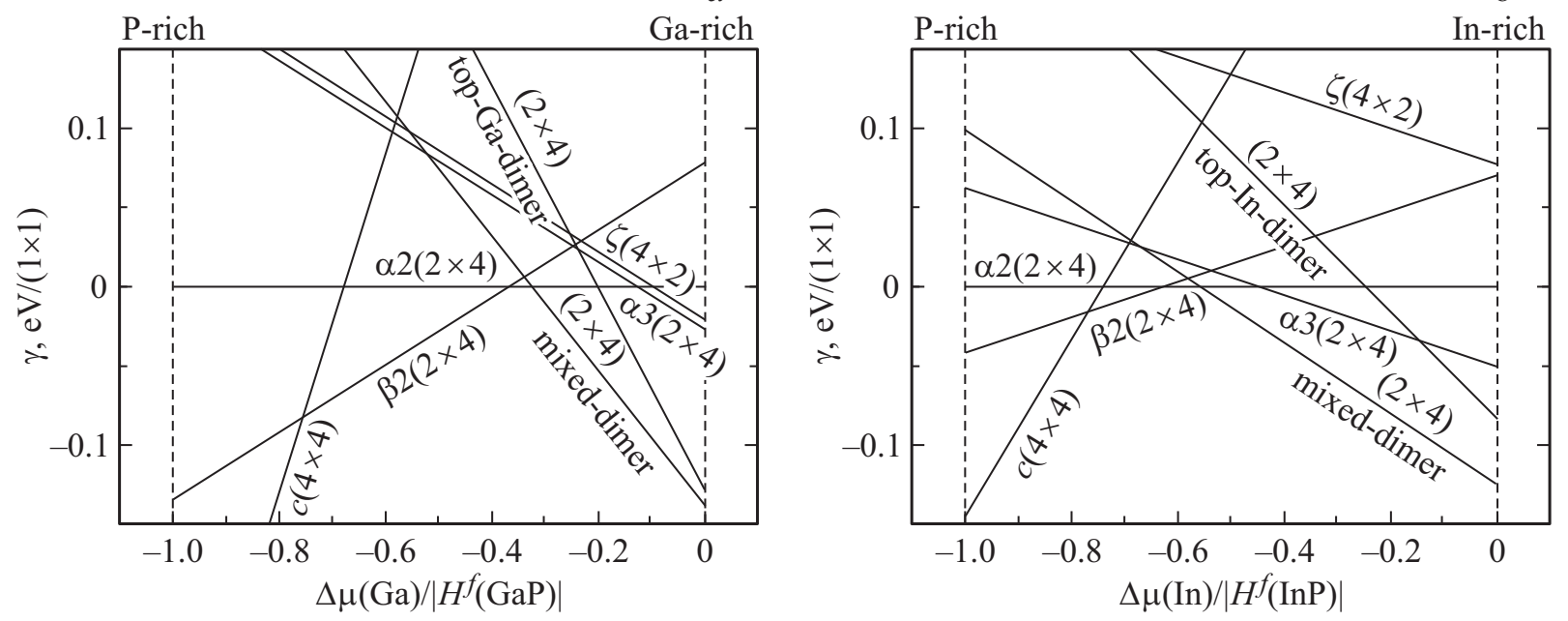

$c$
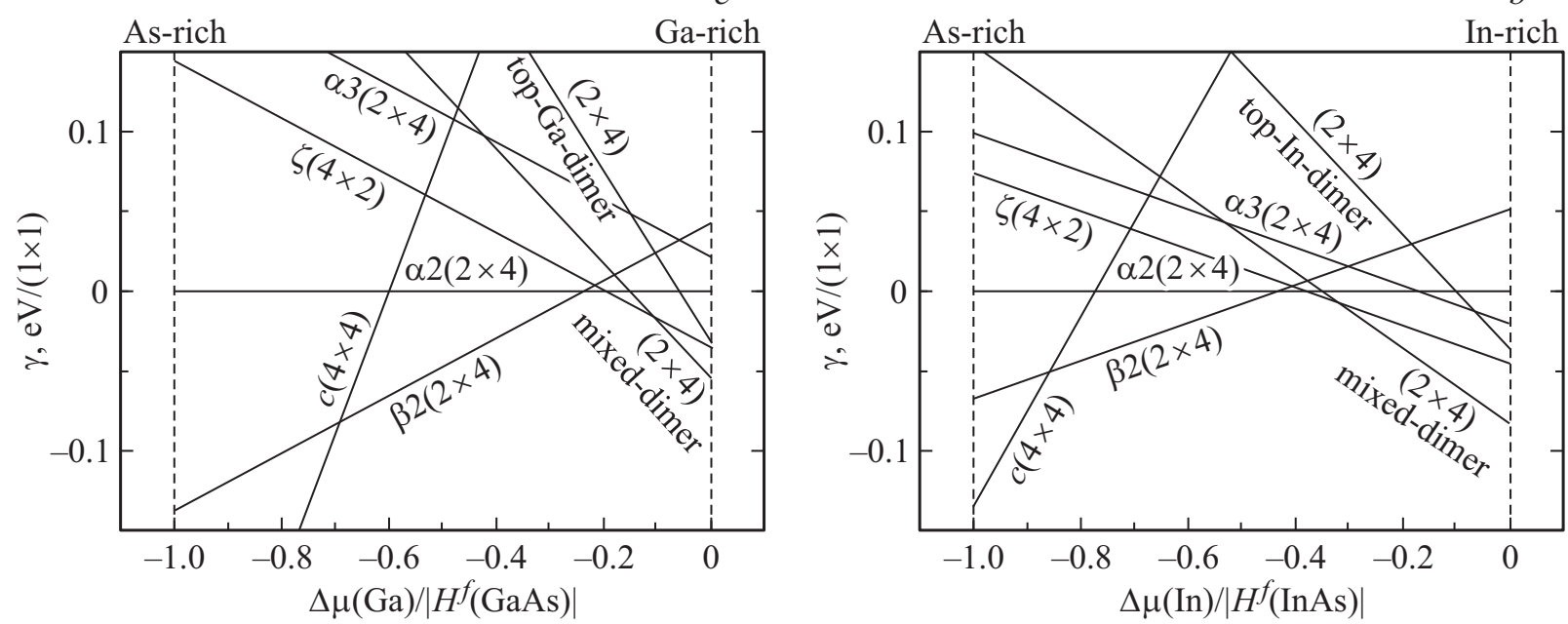

Рис. 4. Зависимость поверхностной энергии в расчете на ячейку $(1 \times 1)$ от химического потенциала галлия (индия) для рассчитанных поверхностных реконструкций $\mathrm{GaP}(a), \operatorname{InP}(b), \operatorname{GaAs}(c)$, InAs $(d)$.

$\mathrm{A}^{\mathrm{III}} \mathrm{B}^{\mathrm{V}}(001)$ с реконструкцией mixed-dimer $(2 \times 4)$ является источником напряжений, поскольку смещение димерных атомов как в плоскости поверхности, так и по нормали к ней зависит от разницы в размерах аниона и катиона. На рис. 5 приведена разница в поверхностных энергиях $(\Delta \gamma)$ для предела обогащения катионами между реконструкциями mixed-dimer $(2 \times 4), \quad \xi(4 \times 2)$ и $\alpha 2(2 \times 4)$ в зависимости от относительной разницы в атомных радиусах катиона и аниона, рассчитанной по следующей формуле: $\Delta r=\left(R_{c}-R_{a}\right) /\left(R_{c}+R_{a}\right)$, где $R_{c}$ и $R_{a}$ - атомные радиусы катиона и аниона соответственно. Положение прямых на рис. 5 определялось методом наименьших квадратов. Видно, что изменение поверхностных энергий реконструкций $\zeta(4 \times 2)$ и $\alpha 2(2 \times 4)$ относительно mixed-dimer $(2 \times 4)$ хорошо коррелирует с $\Delta r$, а коэффициент корреляции достигает значений 0.992 и -0.915 соответственно. В то же время значения $\Delta \gamma$ для $\xi(4 \times 2)$ и $\alpha 2(2 \times 4)$ хорошо коррелируют с $\Delta r$, только если исключить из рассмотрения

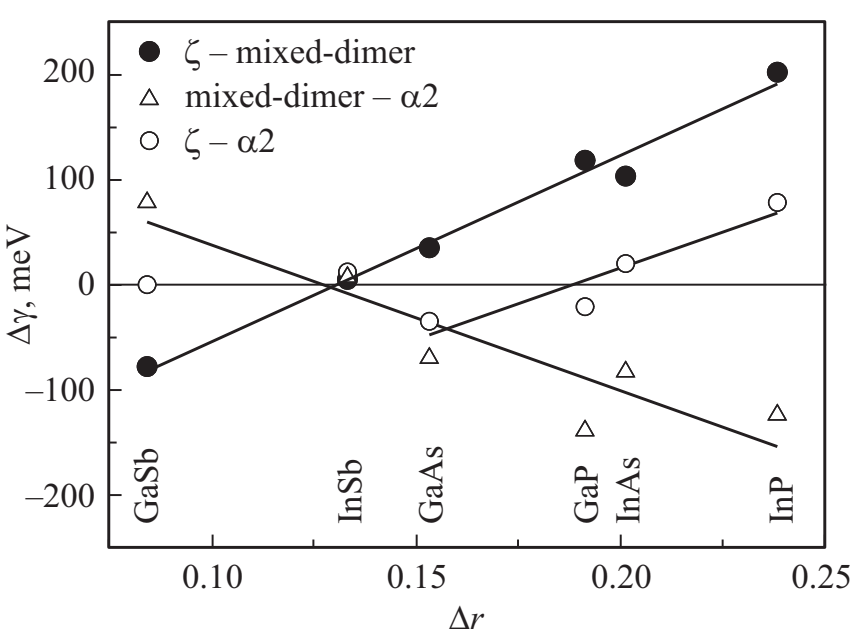

Рис. 5. Разница в поверхностных энергиях реконструкций $\xi(4 \times 2), \alpha 2(2 \times 4)$ и mixed-dimer $(2 \times 4)$ для шести полупроводников $\mathrm{A}^{\mathrm{III}} \mathrm{B}^{\mathrm{V}}$ в зависимости от относительной разницы в атомных радиусах катионов и анионов. 
антимониды галлия и индия, для которых эта разница практически равна нулю. Другими словами, относительное положение прямых, соответствующих реконструкциям $\xi(4 \times 2)$ и $(2 \times 4)$ mixed-dimer, на поверхностных диаграммах стабильности действительно обусловлено разницей в атомных размерах катионов и анионов для шести полупроводников. Это заключение справедливо и для пары реконструкций $\alpha 2(2 \times 4)$ и $(2 \times 4)$ mixed-dimer, тогда как энергетическая предпочтительность $\zeta(4 \times 2)$ относительно $\alpha 2(2 \times 4)$ объясняется размерным эффектом только в случае полупроводников $\mathrm{GaP}, \mathrm{InP}, \mathrm{GaAs}$ и InAs, что требует дальнейших исследований.

\section{3. Электронная структура основных стабильных реконструкций}

Обсудим особенности электронной структуры рассчитанных реконструкций $\alpha(4 \times 3)$ и $\beta(4 \times 3)$ поверхности (001) полупроводников $\mathrm{GaSb}$ и $\mathrm{InSb}$, которая представлена на рис. 6. Положение нуля на рисунке соответствует вершине валентной зоны на проекции объемных состояний. Совмещение электронного спектра поверхности и проекции объемных состояний проводилось по уровню Ферми. Кроме того, на рис. 6 точками показаны поверхностные состояния. Отметим, что под поверхностными состояниями обычно понимаются состояния, локализованные преимущественно на поверхностных атомах, при этом встает вопрос, какие атомы считать поверхностными в случае сложной структуры. На рис. 6 черными точками показаны состояния, локализация которых на атомах димеров превышает 20\%, при этом размер точек указывает на степень локализации. Белые точки показывают состояния, локализованные на атомах, которые расположены ниже димеров и формально не могут считаться поверхностными, однако их координация понижена вследствие образования поверхности. Из рис. 6 видно, что дно зоны проводимости образовано в основном состояниями димеризованных атомов сурьмы, тогда как вершина валентной зоны трехкратно координированными атомами сурьмы.

Напомним, что ранее электронные энергетические спектры поверхности $\mathrm{GaSb}(001)$ с реконструкциями $\alpha(4 \times 3)$ и $\beta(4 \times 3)$ рассчитывались только в работе [21], в которой использовался метод псевдопотенциала, поэтому обсуждение начнем именно с этого полупроводника. В качестве поверхностных состояний в [21] рассматривались состояния, хотя бы частично расположенные в запрещенной щели. В настоящем расчете проекция объемных состояний значительно отличается от проекции из работы [21]. В частности, вершина валентной зоны в точке $\bar{K}$ расположена лишь на 0.33 эВ ниже, чем в точке $\bar{\Gamma}$, тогда в [21] эта разница составляла $\sim 0.83$ эВ. Обратная тенденция наблюдается для дна зоны проводимости: в точке $\bar{K}$ она на 1.29 эВ выше, чем в точке $\bar{\Gamma}$, а в [21] эта разница достигала лишь $\sim 0.38$ эВ. Вследствие данных различий в настоящей работе в запрещенной щели находится большее число незанятых состояний и только несколько занятых состояний. Отметим также, что запрещенная щель в электронном спектре поверхности с $\alpha(4 \times 3)$ и $\beta(4 \times 3)$ на 17.3 и 20.2\% больше, чем на проекции объемных состояний (рис. 6, $a, b$ ). В [21] наблюдалась обратная тенденция, и запрещенная щель в спектре поверхности с этими же реконструкциями была меньше на 26 и 15\% соответственно. Отметим, что обычно (см. работы $[11,16]$ и ссылки в них) при использовании подхода тонких пленок для моделирования поверхности наблюдается именно уширение запрещенной щели в электронном спектре реконструированной поверхности по сравнению с проекцией объемных состояний.

Нижнее незанятое поверхностное состояние $\left(C_{1}\right)$ в случае поверхности $\mathrm{GaSb}(001)-\alpha(4 \times 3)$ обусловлено в основном состояниями, локализованными на димеризованных атомах $\mathrm{Sb}$ (эти состояния обусловлены разрыхляющими $\sigma^{*}$-орбиталями, формирующимися благодаря гибридизации $\mathrm{Ga}-\mathrm{Sb}$, смещенными к атому сурьмы), причем, если вблизи точки $\bar{\Gamma}$ вклад всех димеров примерно одинаковый, то в точке $\bar{K}$ вклад среднего димера (d2 на рис. $1, c)$ становится доминирующим. В последнем случае вклад в поверхностное состояние дает также оборванная связь атома галлия этого димера. В работе [21] единственное незанятое поверхностное состояние $C_{1}$ полностью ассоциировалось с оборванной связью атома галлия среднего димера $d 2$. Следующее незанятое поверхностное состояние $\left(C_{2}\right)$ также обусловлено димеризованными атомами: в точке $\bar{\Gamma}$ основной вклад дают атомы Ga всех димеров, а в точке $\bar{K}-$ атомы $\mathrm{Sb}$ димеров $d 1$ и $d 3$. Вклад в состояние $C_{3}$ в точке $\bar{\Gamma}$ обусловлен главным образом атомами димера $d 3$. Интересно, что занятые поверхностные состояния образуют практически плоские зоны, при этом локализация этих состояний слабо меняется в двумерной зоне Бриллюэна (2D BZ). Так, верхнее занятое поверхностное состояние $\left(V_{1}\right.$ на рис. $\left.6, a\right)$ во всех рассмотренных точках неприводимой части зоны Бриллюэна локализовано на трехкратно координированных атомах $\mathrm{Sb}$ второго слоя, связанных с атомами димеров $d 1$ и $d 3$ (рис. 1,c). Аналогично, состояние $V_{2}$ обусловлено трехкратно координированными атомами $\mathrm{Sb}$ второго слоя, которые образуют связь с атомами димера $d 0$. Отметим, что в работе [21] имела место обратная ситуация: состояние $V_{1}$ было обусловлено атомами сурьмы вблизи димера $d 0$, а $V_{2}$ - атомами сурьмы вблизи $d 1$ и $d 3$.

В случае реконструкции $\beta(4 \times 3)$ поверхности $\mathrm{GaSb}(001)$ электронный спектр выглядит практически аналогично спектру структуры $\alpha(4 \times 3)$. Описанный выше характер локализации поверхностных состояний практически не меняется. Основное отличие заключается в отсутствии вкладов атомов галлия в состояния $C_{1}$ и $C_{2}$, поскольку димеры $d 1-d 3$ образованы только атомами сурьмы. Таким образом, в случае реконструкции $\beta(4 \times 3)$ состояния $C_{1}$ и $C_{2}$ обусловлены только атомами сурьмы димеров $d 1-d 3$. Отметим, что локализация 

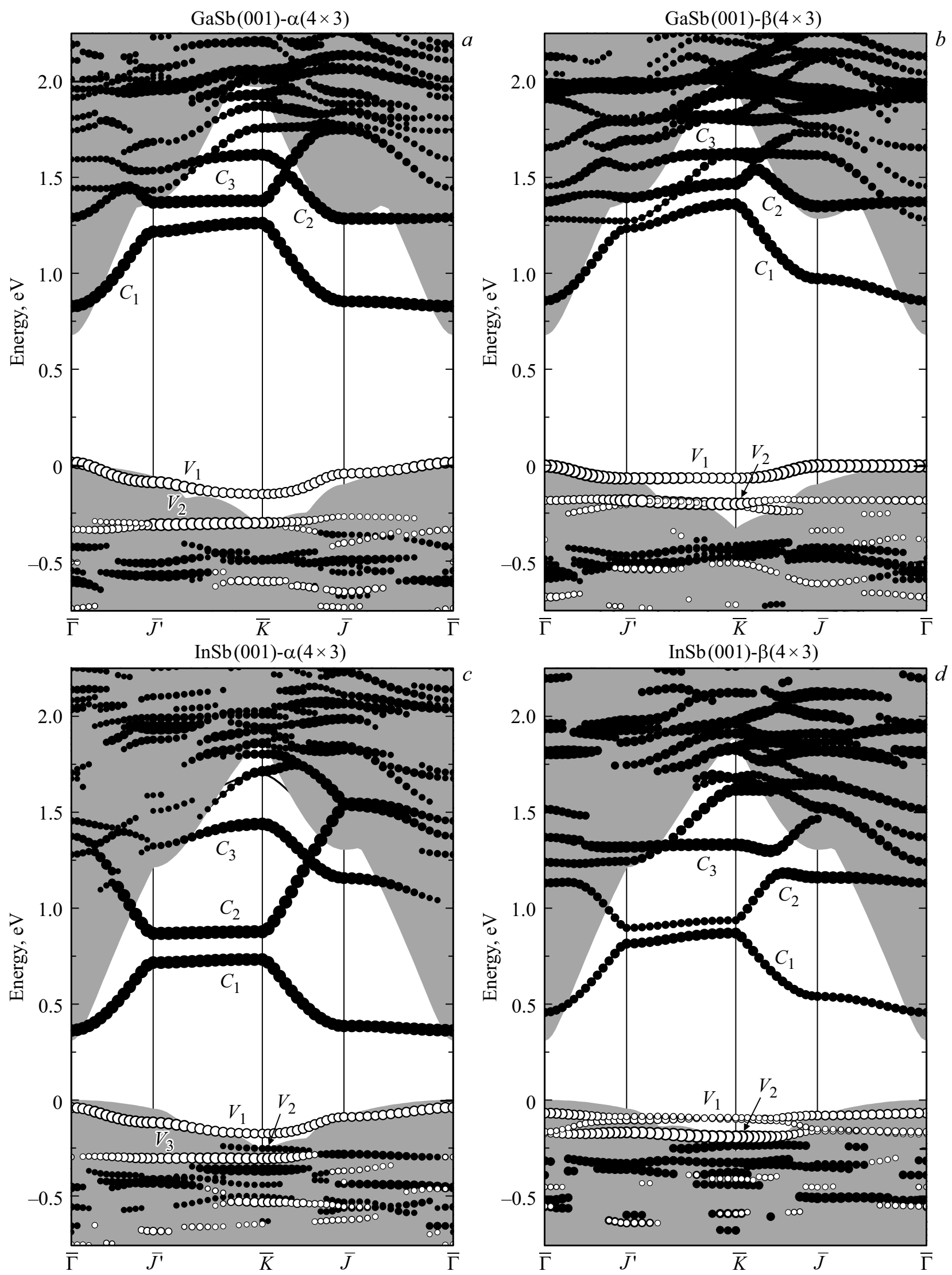

Рис. 6. Электронные энергетические спектры поверхности (001) полупроводников $\mathrm{GaSb}(a, b)$ и $\mathrm{InSb}(c, d)$ с реконструкциями $\alpha(4 \times 3)(a, c)$ и $\beta(4 \times 3)(b, d)$. Положение нуля на энергетической шкале соответствует уровню Ферми и вершине валентной зоны на проекции объемных состояний, показанной серой заливкой. Черными и белыми точками показаны состояния, локализованные на димеризованных атомах и на атомах с уменьшенной координацией соответственно. 
поверхностных состояний для реконструкций $\alpha(4 \times 3)$ и $\beta(4 \times 3)$ в работе [21] была одинаковой.

При замене катиона с $\mathrm{Ga}$ на In также происходят лишь незначительные изменения в электронном спектре реконструкции $\alpha(4 \times 3)$. Поскольку ширина щели в случае полупроводника InSb значительно меньше по сравнению с GaSb, то незанятые поверхностные состояния располагаются ближе к валентной зоне. В частности, состояние $C_{1}$ смещается в сторону валентной зоны на $0.47-0.53$ эВ, $C_{2}-$ на $0.13-0.50$ э $\mathrm{B}, C_{3}-$ на $\sim 0.18$ эВ. Отметим, что сильнее поверхностные состояния смещаются на краю $2 \mathrm{D} \mathrm{BZ}$, тогда как в точке $\bar{\Gamma}$ смещение состояний выражено в меньшей степени. Запрещенная щель в спектрах поверхности $\operatorname{InSb}(001)$ с реконструкциями $\alpha(4 \times 3)$ и $\beta(4 \times 3)$ на 13.6 и $31.1 \%$ больше по сравнению с проекцией объемных состояний. Из рис. $6, c$ видно, что первое и третье занятые состояния $\left(V_{1}\right.$ и $\left.V_{3}\right)$ локализованы на трехкратно координированных атомах $\mathrm{Sb}$, тогда как $V_{2}$ обусловлено димеризованными атомами $\mathrm{Sb}$. Эта незначительная особенность (локализация состояния $V_{2}$ на димеризованных атомах сурьмы) электронного спектра также отличает его от спектра поверхности $\mathrm{GaSb}(001)-\alpha(4 \times 3)$. В то же время спектр поверхности $\operatorname{InSb}(001)$ с реконструкцией $\beta(4 \times 3)$ изменяется в большей степени по сравнению со спектром поверхности $\mathrm{GaSb}(001)$. Так, если состояние $C_{1}$ лишь смещается в сторону валентной зоны на $0.40-0.49$ эВ, то кривая дисперсии $C_{2}$ становится более изогнутой, хотя локализация соответствующих состояний не изменяется по сравнению с $\beta(4 \times 3)-\mathrm{GaSb}(001)$. Таким образом, в случае антимонидов галлия и индия химический состав катионов незначительно влияет на электронную структуру реконструированной поверхности (001).

\section{4. Заключение}

В настоящей работе методом проекционных присоединенных волн в рамках теории функционала электронной плотности проведены расчеты атомной и электронной структуры ряда реконструкций полярной поверхности (001) полупроводников GaSb и InSb. Была рассмотрена группа реконструкций, включающая как характерные для других полупроводников $\mathrm{A}^{\mathrm{III}} \mathrm{B}^{\mathrm{V}}$ поверхностные структуры с симметрией $(2 \times 4),(4 \times 2)$ и $c(4 \times 4)$, так и специфические для антимонидов структуры $(4 \times 3)$. На основе рассчитанных энергий данных реконструкций построены диаграммы стабильности поверхностных структур. Показано, что в отличие от соответствующих диаграмм $\mathrm{GaP}, \mathrm{InP}, \mathrm{GaAs}$, InAs реконструкция смешанного димера $(2 \times 4)$ не является стабильной в пределе обогащения катионами. Из рассмотренных реконструкций $(2 \times 4)$ и $(4 \times 2)$ на поверхности $\mathrm{GaSb}(001)$ и $\operatorname{InSb}(001)$ наименьшую энергию имеют структуры $\beta 2(2 \times 4)$ и $\alpha 2(2 \times 4)$. В пределе обогащения катионами стабильной найдена реконструкция $c(4 \times 4)$ с тремя анионными димерами, что согласуется с данными для других полупроводников $\mathrm{A}^{\mathrm{III}} \mathrm{B}^{\mathrm{V}}$. Принимая во внимание тот факт, что экспериментально на поверхностях $\mathrm{GaSb}(001)$ и $\mathrm{InSb}(001)$ реконструкции с данной симметрией не установлены, при их исключении из рассмотрения стабильными являются структуры $\alpha(4 \times 3)$ и $\beta(4 \times 3)$ для обоих полупроводников, а также $h 0(4 \times 3)$ в случае $\mathrm{GaSb}$. В целом на поверхности $\mathrm{GaSb}(001)$ вблизи стехиометрического состава могут реализовываться обе реконструкции $-\alpha(4 \times 3)$ и $\beta(4 \times 3)$. Проведен детальный анализ электронных спектров для реконструкций $\alpha(4 \times 3)$ и $\beta(4 \times 3)$ поверхности $(001)$ полупроводников $\mathrm{GaSb}$ и InSb. Установлена локализация поверхностных состояний. Показано, что их природа в случае двух полупроводников подобна, а замена катиона приводит лишь к незначительным изменениям в электронном спектре поверхности.

Проведен анализ корреляций между разницей в поверхностных энергиях $\Delta \gamma$ реконструкций смешанного димера $(2 \times 4), \xi(4 \times 2)$ и $\alpha 2(2 \times 4)$ и относительной разницей в атомных радиусах катиона и аниона $\Delta r$. Показано, что поверхностные энергии реконструкций $\xi(4 \times 2)$ и $\alpha 2(2 \times 4)$ относительно реконструкции смешанного димера $(2 \times 4)$ линейно зависят от $\Delta r$. В то же время значение $\Delta \gamma$ для реконструкций $\xi(4 \times 2)$ и $\alpha 2(2 \times 4)$ хорошо коррелируют с $\Delta r$ для фосфидов и арсенидов галлия и индия, тогда как для антимонидов этих элементов данные реконструкции имеют практически одинаковые поверхностные энергии. Таким образом, в настоящей работе показано, что положение прямых, соответствующих реконструкциям $\xi(4 \times 2)$ и смешанного димера $(2 \times 4)$ относительно $\alpha 2(2 \times 4)$ структуры на диаграммах стабильности обусловлено размерным фактором.

\section{Финансирование работы}

Работа выполнена в рамках Программы фундаментальных научных исследований государственных академий наук на 2013-2020 годы, направление III.23.2.8, и программы повышения конкурентоспособности ТГУ. Численные расчеты выполнены на суперкомпьютере SKIF-Cyberia в Томском государственном университете.

\section{Конфликт интересов}

Авторы заявляют об отсутствии конфликта интересов.

\section{Список литературы}

[1] W. Mönch. Semiconductor Surfaces and Interfaces (Berlin, Springer, 2001).

[2] R. Magri, A. Zunger. Phys. Rev. B, 64, 081305 (2001).

[3] R. Magri, A. Zunger. Phys. Rev. B, 65, 165302 (2001).

[4] M.J. Yang, W.J. Moore, B.R. Bennett, B.V. Shanabrook. Electron. Lett., 34, 270 (1998).

[5] G. Klimeck, R. Lake, D.K. Blanks. Phys. Rev. B, 58, 7279 (1998). 
[6] D.Z.-Y. Ting, T.C. McGill. J. Vac. Sci. Technol. B, 14, 2790 (1996).

[7] Z. Zhang, M.G. Lagally. Science, 276, 377 (1997).

[8] L. Däweritz, R. Hey. Surf. Sci., 236, 15 (1990).

[9] Q.-K. Xue, T. Hashizume, T. Sakurai. Prog. Surf. Sci., 56, 1 (1997).

[10] M. Kuball, D.T. Wang, N. Esser, M. Cardona, J. Zegenhagen. Phys. Rev. B, 51, 13880 (1995).

[11] W.G. Schmidt. Appl. Phys. A, 75, 89 (2002).

[12] W.G. Schmidt, F. Bechstedt. Phys. Rev. B, 54, 16742 (1996).

[13] W.G. Schmidt. Appl. Phys. A, 65, 581 (1997).

[14] S.-H. Lee, W. Moritz, M. Scheffler. Phys. Rev. Lett., 85, 3890 (2000).

[15] R.H. Miwa, R. Miotto, A.C. Ferraz, G.P. Srivastava. Phys. Rev. B, 67, 045325 (2003).

[16] A.V. Bakulin, S.E. Kulkova, S.V. Eremeev, O.E. Tereshchenko. Surf. Sci., 615, 97 (2013).

[17] A. Bakulin, A. Ponomarev, S. Kulkova. IOP Conf. Ser.: Mater. Sci. Eng., 77, 012004 (2015).

[18] G.E. Franklin, D.H. Rich, A. Samsavar, E.S. Hirschorn, F.M. Leibsle, T. Miller, T.C. Chiang. Phys. Rev. B, 41, 12619 (1990).

[19] M.T. Sieger, T. Miller, T.C. Chiang. Phys. Rev. B, 52, 8256 (1995).

[20] W. Barvosa-Carter, A.S. Bracker, J.C. Culbertson, B.Z. Nosho, B.V. Shanabrook, L.J. Whitman, H. Kim, N.A. Modine, E. Kaxiras. Phys. Rev. Lett., 84, 4649 (2000).

[21] M.C. Righi, R. Magri, C.M. Bertoni. Phys. Rev. B, 71, 075323 (2005).

[22] J. Houze, S. Kim, S.-G. Kim, S.C. Erwin, L.J. Whitman. Phys. Rev. B, 76, 205303 (2007).

[23] D. Toton, J. He, G. Gory, J.J Kolodziej, S. Godlewski, L. Kantorovich, M. Szymonsk. J. Phys.: Condens. Matter, 22, 265001 (2010).

[24] C. Kumpf, L.D. Marks, D. Ellis, D. Smilgies, E. Landemark, M. Nielsen, R. Feidenhans'l, J. Zegenhagen, O. Bunk, J.H. Zeysing, Y. Su, R.L. Johnson. Phys. Rev. Lett., 86, 3586 (2001).

[25] C. Kumpf, D. Smilgies, E. Landemark, M. Nielsen, R. Feidenhans'1, O. Bunk, J.H. Zeysing, Y. Su, R.L. Johnson, L. Cao, J. Zegenhagen, B.O. Fimland, L.D. Marks, D. Ellis. Phys. Rev. B, 64, 075307 (2001).

[26] J.J. Kolodziej, B. Such, M. Goryl, F. Krok, P. Piatkowski, M. Szymonski. Appl. Surf. Sci., 252, 7614 (2006).

[27] G. Goryl, O. Boelling, S. Godlewski, J.J. Kolodziej, B. Such, M. Szymonski. Surf. Sci., 601, 3605 (2007).

[28] C. Kendrick, G. LeLay, A. Kahn. Phys. Rev. B, 54, 17877 (1996).

[29] D.L. Feldwinn, J.B. Clemens, J. Shen, S.R. Bishop, T.J. Grassman, A.C. Kummel, R. Droopad, M. Passlack. Surf. Sci., 603, 3321 (2009).

[30] P.E. Blöchl. Phys. Rev. B, 50, 17953 (1994).

[31] G. Kresse, D. Joubert. Phys. Rev. B, 59, 1758 (1999).

[32] G. Kresse, J. Hafner. Phys. Rev. B, 47, 558 (1993).

[33] G. Kresse, J. Furthmüller. Comput. Mater. Sci., 6, 15 (1996).

[34] J.P. Perdew, K. Burke, M. Ernzerhof. Phys. Rev. Lett., 77, 3865 (1996).

[35] I. Vurgaftman, J.R. Meyer, L.R. Ram-Mohan. J. Appl. Phys., 89, 5815 (2001).

[36] K. Shiraishi, T. Ito. Jpn. J. Appl. Phys., 37, L1211 (1998).

[37] H.J. Monkhorst, J.D. Pack. Phys. Rev. B, 13, 5188 (1976).

[38] K.H. Xue, J.H. Yuan, L.R.C. Fonseca, X.S. Miao. Comput. Mater. Sci., 153, 493 (2018).
[39] L.G. Ferreira, M. Marques, L.K. Teles. Phys. Rev. B, 78, 125116 (2008)

[40] L.G. Ferreira, M. Marques, L.K. Teles. AIP Advances, 1, 032119 (2011).

[41] R.R. Pela, M. Marques, L.K. Teles. J. Phys.: Condens. Matter, 27, 505502 (2015).

[42] А.В. Бакулин, С.Е. Кулькова. Изв. вузов. Физика, 57, 122 (2014).

[43] L.J. Whitman, P.M. Thibado, S.C. Erwin, B.R. Bennett, B.V. Shanabrook. Phys. Rev. Lett., 79, 693 (1997).

[44] C. Kittel. Introduction to Solid State Physics, 8th edn (Hoboken, USA, John Wiley \& Sons, 2005) p. 50.

[45] B. Paulus, P. Fulde, H. Stoll. Phys. Rev. B, 54, 2556 (1996).

[46] G.S. Rohrer. Structure and Bonding in Crystalline Materials (Cambridge, England, Cambridge University Press, 2001).

[47] G. Henkelman, B.P. Uberuaga, H. Jónsson. J. Chem. Phys., 113, 9901 (2000).

[48] N.A. Gokcen. Bull. of Alloy Phase Diagrams, 10, 11 (1989).

[49] M. Yoshikawa, A. Nakamura, T. Nomura, K. Ishikawa. Jpn. J. Appl. Phys., 35, 1205 (1996).

[50] K.B. Ozanyan, P.J. Parbrook, M. Hopkinson, C.R. Whitehouse, Z. Sobiesierski, D.I. Westwood. J. Appl. Phys., 82, 474 (1997).

[51] V.P. LaBella, Z. Ding, D.W. Bullock, C. Emery, P.M. Thibado. J. Vac. Sci. Technol. A, 18, 1492 (2000).

[52] T. Hashizume, Q.K. Xue, J. Zhou, A. Ichimiya, T. Sakurai. Phys. Rev. Lett., 73, 2208 (1994).

[53] Q.K. Xue, T. Hashizume, A. Ichimiya, T. Ohno, Y. Hasegawa, T. Sakurai. Sci. Rep. RITU, A44, 113 (1997).

Редактор Л.В. Шаронова

\section{First-principles investigation of (001) surface reconstructions of GaSb and InSb semiconductors}

\author{
A.V. Bakulin ${ }^{1,2}$, S.E. Kulkova ${ }^{1,2}$ \\ ${ }^{1}$ Institute of Strength Physics and Materials Science, \\ Siberian Branch of Russian Academy of Sciences, \\ 634055 Tomsk, Russia \\ ${ }^{2}$ National Research Tomsk State University, \\ 634050 Tomsk, Russia
}

Abstract The atomic and electronic structures of reconstructions with $(2 \times 4),(4 \times 2), c(4 \times 4)$ and $(4 \times 3)$ symmetry on the (001) surface of $\mathrm{GaSb}$ and $\mathrm{InSb}$ semiconductors were studied by the projector augmented-wave method. It was shown that in the cation-rich limit the $\beta 2(2 \times 4)$ reconstruction is stable on the $\mathrm{GaSb}(001)$ surface, whereas $\alpha 2(2 \times 4)$ has the lowest energy in the case of InSb. The $c(4 \times 4)$ reconstruction with three $\mathrm{Sb}$ dimers is found to be stable in the Sb-rich limit. Near stoichiometric composition the $\alpha(4 \times 3)$ and $\beta(4 \times 3)$ structures are stable that is in agreement with experimental data. Electronic structure of the $(4 \times 3)$ reconstructions with lowest surface energy is discussed. A weak influence of chemical composition of cations on the surface state structure and their localization at the formation of $(4 \times 3)$ structures was revealed. The correlation between the surface energy of some $(4 \times 2)$ and $(2 \times 4)$ reconstructions and the difference in the atomic radii of anions and cations was established. 